\title{
PLANT ELECTRICAL ACTIVITY ANALYSIS FOR OZONE POLLUTION CRITICAL LEVEL DETECTION
}

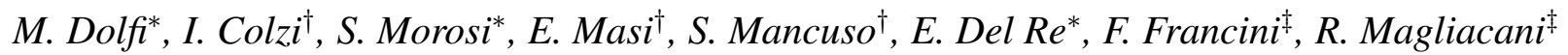 \\ * CNIT - DINFO, University of Florence, via S. Marta 3, 50139 Florence, Italy \\ ${ }^{\dagger}$ DISPAA, University of Florence, viale delle Idee 30, 50019 Sesto Fiorentino, Florence, Italy \\ $\ddagger$ ETG s.r.l., Via di Porto 159, 50018 Scandicci, Florence, Italy
}

\begin{abstract}
The electrical activity signals in plants can provide useful information to monitor environmental conditions, such as atmospheric pollution. Nonetheless the study of the relationship between environmental stimuli and electrical responses of plants is still a critical step in developing technologies that use plants as organic sensing devices. In this paper an automatic method of analysis of plant electrical signals for ozone critical levels detection is proposed, based on the fundamentals of correlation theory. In order to classify the morphology characteristics of plant response to ozone exposure we used a segmentation of time series measurements of the electrical activity of plants before, during and after the stimulation. Then, we extracted the significant deviations from the baseline trend to detect and identify the response to a known stimulus, in terms of correlation coefficient. As a result, the proposed detection algorithm represents a novel monitoring method for detecting critical levels of ozone concentrations.
\end{abstract}

Index Terms - Plant electrical signal, ozone pollution, spike detection, waveform correlation, data classification

\section{INTRODUCTION}

Atmospheric pollution has become one of the most serious environmental problems of the modern world. Its adverse effects are associated with the degradation of the quality of life, affecting the sustainability of urban ecosystems [1]. The problem of the worsening air quality in highly anthropized environments exerts nowadays a high level of interest within the scientific community and public opinion because of the known strong relationship between exposure to many air pollutants and increased adverse effects on human health [2-4]. Among air pollutants, ozone is one of the most important greenhouse gas [5] with secondary origin, generated in the troposphere through a series of complex photochemical reactions involving solar radiation and ozone precursors, i.e. methane $\left(\mathrm{CH}_{4}\right)$, carbon monoxide $(\mathrm{CO})$, volatile organic compounds (VOCs), and nitrogen oxides $\left(\mathrm{NO}_{\mathrm{x}}\right)$, which are largely emitted from anthropogenic sources [6]. Background $\mathrm{O}_{3}$ concentrations have risen from $\sim 10 \mathrm{ppb}$ before the in- dustrial revolution [7] to daytime summer concentrations exceeding $40 \mathrm{ppb}$ in many parts of the Northern Hemisphere [8]. Due to its nature of reactive oxidant agent, ozone can generate several negative effects on human health including lung inflammation, reduced lung function, degenerative diseases, age related disorders and eventually cancer [9]. Ozone also acts as a corrosive agent for many materials, surface coatings and buildings [10]. Therefore, it is easy to understand the importance of a proper air quality management and of the attention to new reliable approaches for ozone monitoring, such as the use of plant as biosensors. The most common air quality measurements exploit sensors based on the use of physicochemical properties in order to measure the concentrations of air pollutants. In comparison with the traditional monitoring systems, the use of biosensors has the advantage to show us the real pollutants impact on living organisms, thus providing additional data to the electronic instruments. Moreover, this allows to take into account the concepts of bioavailability, dose and exposure, resulting in a more realistic approach of the pollutants impact on environmental and human health [11]. An ideal monitoring system should be biologically-based and at the same time practical for wide use. Plants perfectly reflect this feature, being naturally widespread in our environment, easy and cheap to product and to maintain thanks to their self-sustainability. Moreover, plants are more sensitive than humans and animals in terms of physiological reaction to fluctuations of multiple parameters [12]. Because of their sessile nature, plants are indeed continuously exposed to a wide variety of environmental changes to which they are able to respond by adjusting their physiological characteristics to limit possible damages. These remarkable characteristics make plants suitable tools for environmental monitoring. The advantage of this kind of bio-monitoring is to allow the follow-up of air quality evolution and the extent of its impact on vast zones at low cost. On the other hand, the interpretation of the results could be made difficult by the influence of other environmental parameters and of the ecosystems heterogeneity, requiring the participation of specialists [11]. Moreover, this kind of analysis can give us just long-term exposure information. In the present study, we propose a new approach to use plants as 
easy and dynamic bio-sensors able to provide real-time data on air quality changes, particularly referring to ozone concentration. Ozone effect on plants determines changes in growth and appearance of visible symptoms (e.g. chlorosis, necrosis) but this response is preceded by a series of biochemical events, the so-called "hidden injury" [13]. All these changes at physiological level are reflected in the generation of electrical signals. It is known from time that plants produce electrical signals when subjected to various environmental stimuli [14-18]. These electrical signals in essence represent changes in underlying physiological processes influenced by the external stimuli. Since plants react to environmental changes generating responses in the bioelectrical activity, this lead to the possibility to classify external stimuli from the typical electrical signal response [18]. The focus of our work was to find an association between ozone exposure and some typical features in the resulting plant electrical signal, in order to create a classification algorithm able to identify the stimulus. In order to obtain reliable results, automatic response detection and data classification for plant electrical signals are necessary to be developed. Many papers reported artifacts detection methods for EEG and EKG analysis [19-23]. Various advanced methods have been applied to detect artifacts in EEG signals, such as independent component analysis (ICA) [19-21], support vector machine (SVM) [19], wavelet analysis [22] and autoregressive (AR) model [23]. These methods were appropriate for human biological signals and offline analysis. For the analysis of plants bio-electrical signals related to environmental changes the response detection algorithm needs to be simplified. In this paper a correlation based data classification system for plant electrical signal analysis is proposed. A dataset of electrical signals was collected from ligustrum and buxus plants exposed to ozone in controlled conditions. These species have been selected for the study because of their widespread use in urban sites. To automatically segment the signals a derivative-based detection method was designed, similarly to those used in spike detection [24]. Finally, the detected signals were classified based on correlation waveform analysis of plant response to ozone air pollution. The proposed data classification method can be extended for various research purposes by defining weight coefficients and adjusting thresholds.

\section{DATA ACQUISITION}

The experiments were performed inside a closed growth chamber, the so-called iTreeBox, in order to control the ozone concentration and the other environmental parameters. Inside the box plants were exposed to standard artificial light conditions by means of LED lights responding to the plants photosynthetic needs (PAR radiation). About $50 \mathrm{~cm}$ high plants of Ligustrum texanum and Buxus macrophilla were used for the experiments and each plant was placed in the box and exposed to ozone stimulus one at a time. Electrical sig-

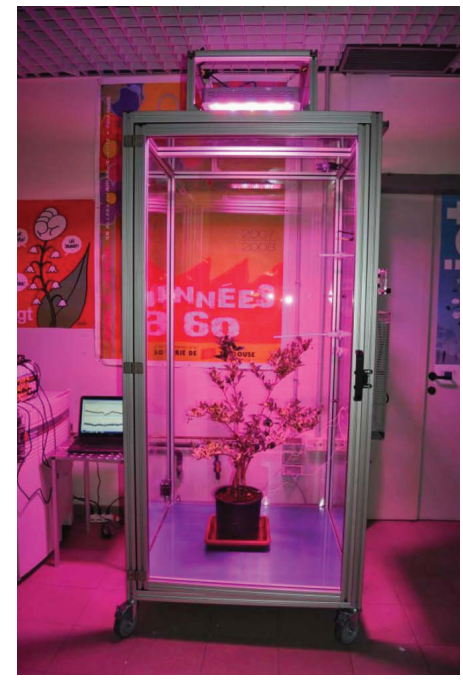

Fig. 1. The iTreeBox plant growth chamber

nals were monitored by means of three stainless steel needle electrodes, one placed at the base (reference for background noise subtraction), one in the middle and the other on top of the stem. After some preliminary test, the sampling frequency was set as 10 samples/ $s$ for all the recordings. All the experiments were carried out during the day time for about 8 hours and the ozone treatment always started at least one hour after the beginning of the electrical signal acquisition to allow the plant acclimating to the artificial light and the box conditions. Before exposing plants to the pollutant, several acquisitions in natural conditions (without ozone stimulus) were performed, in order to monitor the physiological electrical activity of each plant. The ozone treatment consisted of one or two expositions (at a time distance of two hours) to a constant concentration of $200 \mathrm{ppb}$ for 60 minutes. Moreover, further experiments consisted in exposing the plant to a gradual increase of ozone concentration, to simulate a more realistic environmental condition of summer heat days. The ozone was injected in the box at different increasing concentrations every 60 minutes for a total duration of the experiment of 4 hours. More specifically, ozone concentrations used were $50,100,150$ and $200 \mathrm{ppb}$, each of which was maintained constant inside the box for 60 minutes.

\section{DATA ANALYSIS}

The proposed detection algorithm of plant response to ozone is designed according to two approaches. The first is based on a preliminary extraction of significant deviations from a certain baseline trend: in order to correctly identify the response in an automatic way, a derivative-based algorithm has been used. The second is based on the classification of the ozone risk level by the method of correlation. In all applications we used the signals from the experiments carried out in the 


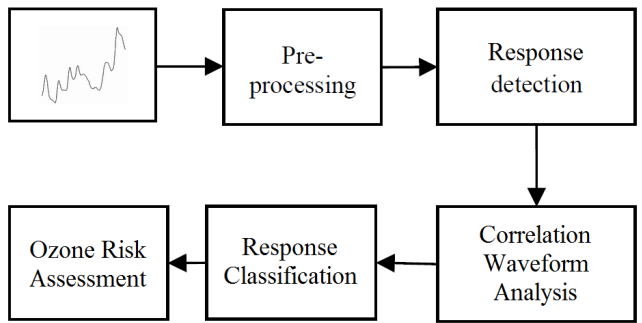

Fig. 2. Flow chart of the detection algorithm

iTreeBox chamber. The methods were developed under Matlab software. The detailed flow chart of the proposed system is shown in Figure 2.

\subsection{Pre-processing of the plant electrical signal}

The reference signals generated by a plant are generally contaminated by different sources of noise. Since most of the energy of such biological signals is concentrated at low frequencies, we applied a low-pass filter, followed in cascade by a moving average filter to further clean the signal. Given the fact that the responses to an ozone stimulus last approximately 60 minutes, the used low-pass filter has a cutoff frequency of $5 \mathrm{mHz}$.

\subsection{Plant response detection}

In general, in response to an environmental stimulus, the plant electrical activity appears irregular for a certain time window. We use the different characteristics induced by ozone air pollution to detect the abnormal signal waveform. In order to automatically segment the data and correctly identify the response, we implemented a derivative-based algorithm. Given the voltage signal $V(t)$ and the following parameters vector:

$$
P=\left(A_{d V}, \Delta t_{d}, S_{V}\right)
$$

a response is defined to occur when the first derivative of the signal decreases below a negative threshold $A_{d V}$ :

$$
\frac{d V(t)}{d t}<A_{d V}
$$

In order not to associate very quick fluctuations to actual responses, we set another threshold, $\Delta t_{d}$, as a minimum time duration following the onset of the response. This condition enables the accurate detection of long-lasting effects on the plant electrical activity caused by ozone exposure. Based on the supplied data, it has been noticed that the central position of the response is related to the nearest local minimum of the plant voltage signal: if the response voltage initially decreases, after a certain time period it will start to increase in order to restore the pre-stimulation baseline trend. In our approach, the period taken for the plant to stabilize its potential after the stimulus has to be assigned to the same response.

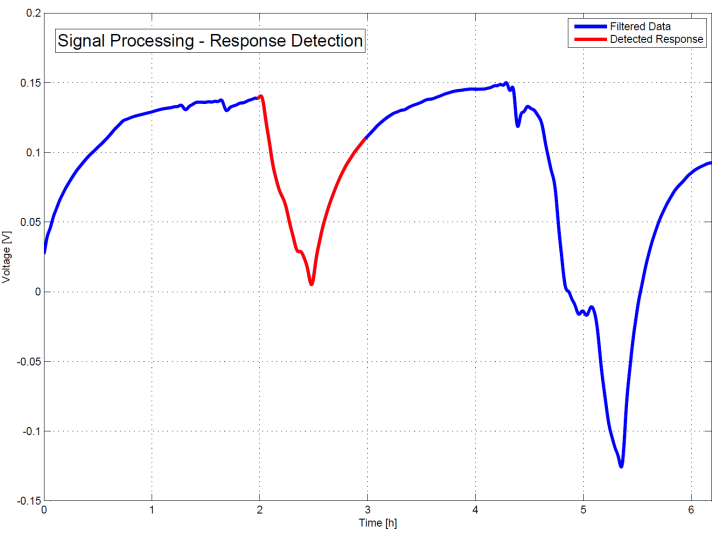

Fig. 3. Response detection of ligustrum plant signal after ozone exposure

This property was used to estimate the minimum variation in the slopes of the ozone response and set an amplitude threshold, $S_{V}$, on the voltage signal. The ozone response is then detected and extracted whenever the difference between the central location of the response, $V_{c}$, and the basal voltage $V_{b}$, that is the value of the voltage signal preceding the onset of response, exceeds the threshold $S_{V}$ :

$$
\left|V_{c}-V_{b}\right|>S_{V}
$$

An example of detected ozone response is depicted in Figure 3. A representative ozone response template, constructed by coherent averaging of the respective response segments of the recordings used for the training phase, was employed for subsequent comparison with all the responses detected by the proposed system. A window size of 60 minutes was used, in order to effectively include the long-lasting repolarization phase of the plant signal.

\section{CORRELATION WAVEFORM ANALYSIS FOR OZONE RESPONSE CLASSIFICATION}

Cross correlation is a statistical technique which can show whether and how strongly pairs of variables are related. It is an excellent tool to match images and signals with each other. It is robust to noise, and can be normalized for pattern matching. The correlation coefficient, a statistical measure of similarity of two waveforms, produces a value, $\rho$, which falls within the range $[-1,+1]$, where +1 indicates a perfect match between signal and template. Mathematically, the correlation coefficient is defined as follows:

$$
\rho=\frac{\sum_{i=1}^{N}\left(t_{i}-\bar{t}\right)\left(s_{i}-\bar{s}\right)}{\sqrt{\sum_{i=1}^{N}\left(t_{i}-\bar{t}\right)^{2}} \sqrt{\sum_{i=1}^{N}\left(s_{i}-\bar{s}\right)^{2}}}
$$

where $t_{i}$ are the template points, $s_{i}$ are the signal points under analysis, $\bar{t}$ is the average value of the template points, $\bar{s}$ is the 
average value of the signal points, $N$ is the number of points in the template, and $\rho$ is the performance measure. The correlation coefficient is independent of the relative amplitudes of two signals and independent of any baseline changes. Based on the supplied data, it was observed that the plant response to ozone stimulus is characterized by a specific waveform. The proposed detection system takes advantage of this property to classify the risk level of ozone air pollution by using the correlation coefficient. Several studies have offered guidelines for the interpretation of the size of a correlation. The interpretation of the correlation coefficient depends on the context and purposes. In our study an empirical approach was adopted, by giving numerous plant signals to the system in order to adjust and validate the detection and classification threshold values of the proposed algorithm. The correlation-based classifier has been implemented to distinguish electrical responses to critical levels of ozone exposure by identifying the detected responses with very strong correlation to the template.

\section{EXPERIMENTAL RESULTS}

To examine the efficiency of the algorithms, a database of 84 day-long recordings of plant electrical activity was employed. The recordings were chosen to include a broad variety of waveform responses. The database was collected from both ligustrum and buxus plants, including experiments carried out with various levels of exposure to ozone air pollution and in natural conditions. The correctness of a classification can be evaluated by computing the number of correctly recognized class examples (true positives, $t p$ ), the number of correctly recognized examples that do not belong to the class (true negatives, $t n$ ), and examples that either were incorrectly assigned to the class (false positives, $f p$ ) or that were not recognized as class examples (false negatives, $f n$ ). According to [25], the following performance measures for classification are considered:

$$
\begin{aligned}
\text { Accuracy } & =\frac{t p+t n}{t p+f n+f p+t n} \\
\text { Precision } & =\frac{t p}{t p+f p} \\
\text { Sensitivity } & =\frac{t p}{t p+f n} \\
\text { Specificity } & =\frac{t n}{f p+t n}
\end{aligned}
$$

The detection results of the proposed algorithm are listed in Table 1. The classification system is shown to be capable of discriminating the response to critical levels of ozone air pollution from the depolarizations induced by effects of natural environmental conditions with $87 \%$ accuracy. However, individual thresholds were required for each plant species and were based on the initial training phase. The total performance is high since the achieved precision and specificity are high for the ligustrum plant dataset $(96 \%$ and $95 \%$ respectively), compared to the results of the buxus plant dataset ( $89 \%$ precision and $77 \%$ sensitivity). The main advantage of the proposed system resides in the fact that the classification algorithm based on correlation coefficient, by recognizing the degree of similarity between plant electrical signal and template waveform provides a very efficient and innovative monitoring technology for detecting ground-level ozone pollution.

Table 1. Results from the classification algorithm

\begin{tabular}{lccc}
\hline & ligustrum & buxus & Total Performance \\
\hline Accuracy & $92 \%$ & $81 \%$ & $87 \%$ \\
Precision & $96 \%$ & $89 \%$ & $93 \%$ \\
Sensitivity & $89 \%$ & $77 \%$ & $84 \%$ \\
Specificity & $95 \%$ & $85 \%$ & $91 \%$ \\
\hline
\end{tabular}

\section{CONCLUSIONS}

In this paper has been presented an automatic method of analysis of plant electrical signal in order to detect critical levels of ozone air pollution. The experimental data were coming from plants exposed to various ozone concentrations in a closed plant growth chamber, specifically designed to recreate typical environmental and daylighting conditions. The proposed classification algorithm is based on the correlation theory; it mainly recognizes the degree of similarity between a reference ozone response and the acquired plant electrical signal. Then the decision is made based on the correlation coefficient. The experimental results show that the proposed system achieve over all accuracy of $87 \%$. Moreover the innovative approach to the problem of atmospheric pollution monitoring, based on plant electrical activity analysis, allows the classifier to be easily extended to other major air pollutant classes in future studies.

\section{REFERENCES}

[1] Chen W. Y. Jim C.Y., "Assessing the ecosystem service of air pollutant removal by urban trees in Guangzhou (China)," Journal of Environmental Management, vol. 88, pp. 665-676, September 2008.

[2] Robert L. Maynard, "Chapter 6 Health Effects of Urban Pollution," in Air Quality in Urban Environments, vol. 28, pp. 108-128. The Royal Society of Chemistry, 2009.

[3] Robert J. Laumbach and Howard M. Kipen, "Respiratory health effects of air pollution: Update on biomass smoke and traffic pollution," Journal of Allergy and Clinical Immunology, vol. 129, no. 1, pp. 3 - 11, 2012.

[4] Mauro Masiol, Claudio Agostinelli, Gianni Formenton, Enzo Tarabotti, and Bruno Pavoni, "Thirteen years of 
air pollution hourly monitoring in a large city: potential sources, trends, cycles and effects of car-free days," The Science of the total environment, vol. 494-495, pp. 8496, October 2014.

[5] Arlene M. Fiore, Daniel J. Jacob, Brendan D. Field, David G. Streets, Suneeta D. Fernandes, and Carey Jang, "Linking ozone pollution and climate change: The case for controlling methane," Geophysical Research Letters, vol. 29, no. 19, pp. 25-1-25-4, 2002.

[6] Elena Paoletti, Alessandra De Marco, David C.S. Beddows, Roy M. Harrison, and William J. Manning, "Ozone levels in European and USA cities are increasing more than at rural sites, while peak values are decreasing," Environmental Pollution, vol. 192, no. 0, pp. $295-299,2014$

[7] A. VOLZ and D. KLEY, Evaluation of the Montsouris series of ozone measurements made in the nineteenth century, 1988.

[8] R. Vingarzan, "A review of surface ozone background levels and trends," Atmospheric Environment, vol. 38, pp. 3431-3442, 2004.

[9] Marilena Kampa and Elias Castanas, "Human health effects of air pollution," Environmental Pollution, vol. 151, no. 2, pp. $362-367$, 2008, Proceedings of the 4th International Workshop on Biomonitoring of Atmospheric Pollution (With Emphasis on Trace Elements).

[10] M. Escudero, A. Lozano, J. Hierro, J. d. Valle, and E. Mantilla, "Urban influence on increasing ozone concentrations in a characteristic Mediterranean agglomeration," Atmospheric Environment, vol. 99, pp. 322-332, Dec. 2014

[11] Jaro Falla, Philippe Laval-Gilly, Michel Henryon, Dominique Morlot, and Jean-franois Ferard, "Biological Air Quality Monitoring: a Review," Environmental Monitoring and Assessment, vol. 64, no. 3, pp. 627644,2000

[12] Tatiana Wuytack, Kris Verheyen, Karen Wuyts, Fatemeh Kardel, Sandy Adriaenssens, and Roeland Samson, "The potential of biomonitoring of air quality using leaf characteristics of white willow (Salix alba L.)," Environmental Monitoring and Assessment, vol. 171, no. 1-4, pp. 197-204, 2010

[13] Jenny Renaut, Sacha Bohler, Jean-Franois Hausman, Lucien Hoffmann, Kjell Sergeant, Nagib Ahsan, Yves Jolivet, and Pierre Dizengremel, "The impact of atmospheric composition on plants: A case study of ozone and poplar," Mass Spectrometry Reviews, vol. 28, no. 3, pp. 495-516, 2009.

[14] J. Burdon Sanderson, "Note on the Electrical Phenomena Which Accompany Irritation of the Leaf of Dionaea muscipula," Proceedings of the Royal Society of Lon- don, vol. 21, no. 139-147, pp. 495-496, 1872.

[15] BarbaraG. Pickard, "Action potentials in higher plants," The Botanical Review, vol. 39, no. 2, pp. 172-201, 1973.

[16] Eric Davies, "Electrical Signals in Plants: Facts and Hypotheses," in Plant Electrophysiology, AlexanderG. Volkov, Ed., pp. 407-422. Springer Berlin Heidelberg, 2006.

[17] JRG FROMM and SILKE LAUTNER, "Electrical signals and their physiological significance in plants," Plant, Cell and Environment, vol. 30, no. 3, pp. 249257, 2007.

[18] S. K. Chatterjee, S. Ghosh, S. Das, V. Manzella, A. Vitaletti, E. Masi, L. Santopolo, S. Mancuso, and K. Maharatna, "Forward and Inverse Modelling Approaches for Prediction of Light Stimulus from Electrophysiological Response in Plants," ArXiv e-prints, Oct. 2014.

[19] JunFeng Gao, Yong Yang, Pan Lin, Pei Wang, and ChongXun Zheng, "Automatic Removal of EyeMovement and Blink Artifacts from EEG Signals," Brain Topography, vol. 23, no. 1, pp. 105-114, 2010.

[20] P. LeVan, E. Urrestarazu, and J. Gotman, “A system for automatic artifact removal in ictal scalp EEG based on independent component analysis and Bayesian classification," Clinical Neurophysiology, vol. 117, no. 4, pp. 912 - 927, 2006.

[21] Arnaud Delorme, Terrence Sejnowski, and Scott Makeig, "Enhanced detection of artifacts in EEG data using higher-order statistics and independent component analysis," NeuroImage, vol. 34, no. 4, pp. 1443 - 1449, 2007.

[22] Joe-Air Jiang, Chih-Feng Chao, Ming-Jang Chiu, RenGuey Lee, Chwan-Lu Tseng, and Robert Lin, "An automatic analysis method for detecting and eliminating ECG artifacts in EEG," Computers in Biology and Medicine, vol. 37, no. 11, pp. 1660 - 1671, 2007.

[23] Maarten van de Velde, I.Robert Ghosh, and Pierre J.M. Cluitmans, "Context related artefact detection in prolonged EEG recordings," Computer Methods and Programs in Biomedicine, vol. 60, no. 3, pp. 183 - 196, 1999.

[24] Scott B. Wilson and Ronald Emerson, "Spike detection: a review and comparison of algorithms," Clinical Neurophysiology, vol. 113, no. 12, pp. 1873 - 1881, 2002.

[25] Marina Sokolova and Guy Lapalme, "A systematic analysis of performance measures for classification tasks," Information Processing and Management, vol. 45, no. 4, pp. 427 - 437, 2009. 